\title{
Testing the $\mu(I)$ granular rheology against experimental silo data
}

\author{
Luke Fullard $^{1, \star}$, Eric Breard ${ }^{2}$, Clive Davies ${ }^{3}$, Pierre-Yves Lagrée ${ }^{4}$, Stéphane Popinet ${ }^{4}$, and Gert Lube $^{2}$ \\ ${ }^{1}$ Institute of Fundamental Sciences, Massey University, New Zealand \\ ${ }^{2}$ Volcanic Risk Solutions, Massey University, New Zealand \\ ${ }^{3}$ School of Engineering and Advanced technology, Massey University, New Zealand \\ ${ }^{4}$ Institut Jean le Rond d'Alembert, Université Pierre et Marie Curie, Paris, France
}

\begin{abstract}
Industrial storage of granular material using silos is common, however, improved understanding of silo flow is needed. Various continuum models attempt to describe the velocity of dense granular flow in silos. Kinematic, and recently, stochastic models, based upon the diffusion of some quantity, perform well when there is a single orifice, and when the yield criterion is satisfied. However, if system stresses are insufficient to satisfy the yield criterion, or if there is a second orifice, these models fail to capture the entire flow behaviour. Advances in granular rheology have allowed a pressure dependent friction law to be defined which can capture the behaviour of granular silo flow including un-yielded zones, flow-rate independence of fill height, the Beverloo flow-rate, and various other phenomena. We performed silo discharge experiments in a flat bottomed planar silo with a single and two adjacent orifices, for two grain types. The velocity was measured using Particle Image Velocimetry. Results were compared to a mathematical model based on the $\mu(I)$ rheology which was shown to qualitatively capture the observed phenomena including plug-like zones where the yield criterion is not satisfied. These preliminary results strongly encourage future investigations into the effect of friction parameters and numerical boundary conditions.
\end{abstract}

\section{Introduction}

Although granular material has been stored in silo constructions for thousands of years [1] it is only in the last sixty years that significant progress has been made to understand the physics of silo discharge. The mass flow rate of grains from slot and circular orifices is well described using the Beverloo scaling [2, 3], while the velocity field during silo discharge has been described by kinematictype models [4-9], stochastic diffusion models [10, 11], discrete modelling [12-14], and more recently, by utilising a pressure dependent viscosity in a Navier-Stokes solver $[15,16]$. Experimental studies on silo flow have been conducted using 2D imaging techniques such as particle image velocimetry (PIV) or Lagrangian particle tracking [4, 17-19]. These studies have shown that the velocity field near the orifice of a silo is Gaussian-like (which motivated the use of diffusion-like equations to describe some granular silo flows) and mainly radial, but further from the orifice the flow is more plug-like with neighbouring particles flowing at very similar velocities. Although discrete models have been shown to capture most of the mentioned phenomena, the computational overhead is considered too high for many practical industrial flows, particularly when the particle size is small and the silo is large. As such, continuum models have been pursued to model many industrial granular flows including segregation [20], mixing
[6, 21], and blending [22]. Kinematic and stochastic based models describe the Gaussian-like velocity close to the silo orifice well, yet are not predictive of the flow rate, but rather take the flow rate as an input parameter (either explicitly or via a specified velocity far from the orifice). As such, they are unable to describe the Beverloo scaling of flow rate with orifice diameter explicitly, or capture the flow-rate interference of two near-by orifices [23]. It has also been reported that the "diffusion length" parameter in kinematic models is a function of the height in the particle bed [4], which has not been described well by kinematic models. Additionally, since the yield-criterion is assumed satisfied everywhere for these two models, they are unable to describe accurately plug-like flow zones in tall silos. Conversely, models where the so-called $\mu(I)$ rheology [24] is used to define an artificial viscosity in a Navier-Stokes code $[15,16,25]$ have proven to capture all these observed phenomena, although comparison to experimental data is still a work in progress. To this end, we present preliminary results of 2D silo drainage experiments with amaranth grains and mustard seeds and compare the observed results with those derived using the $\mu(I)$ model. The experiments (and numerical tests) are performed with a single centrally located slot orifice, and with two adjacent orifices.

\footnotetext{
^e-mail: L.Fullard@Massey.ac.nz
} 


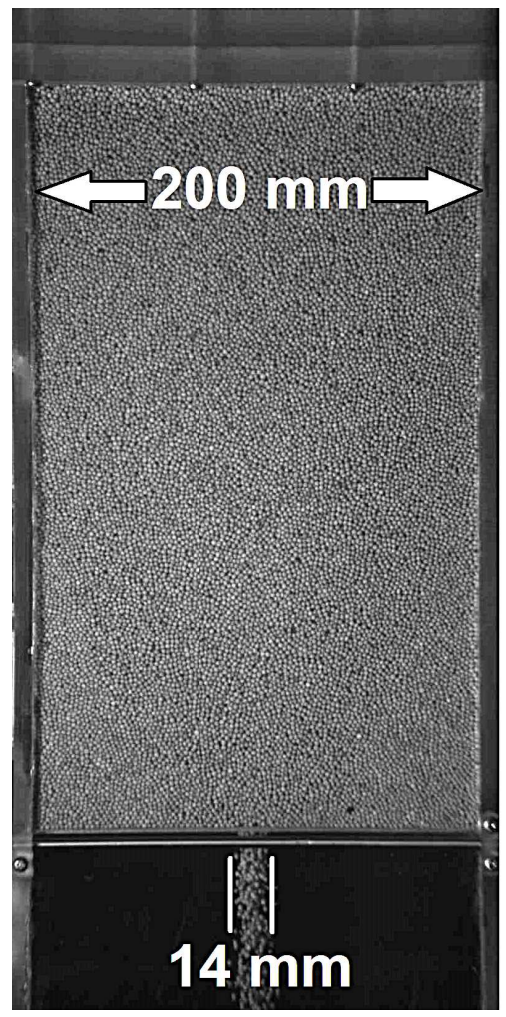

Figure 1. A formatted image showing the silo test area during an experiment with mustard seed and a centrally located exit orifice.

\section{Experimental method and results}

Our experimental system is a flat bottomed rectangular silo constructed from perspex on all sides. The silo has width $200 \mathrm{~mm}$, depth $15 \mathrm{~mm}$ and height $350 \mathrm{~mm}$, and has a groove near the bottom where we can control the orifice size, geometry, and spacing. The silo is filled with either amaranth grains or mustard seeds and the flow is initiated using a sliding bar mechanism. The flow is recorded using a highspeed camera at 340 frames per second (with the exception of the "Single slot: Amaranthus experiment", which was recorded at 500 FPS), and the resulting images are passed through a particle image velocimetry software, PIVLab $[26,27]$, to obtain velocity vectors. The experiments are performed once with a single centrally located rectangular slot orifice, and once with adjacent orifices separated by a distance of $80 \mathrm{~mm}$. The amaranth grains were approximately $1 \mathrm{~mm}$ in diameter, each orifice had a width of $8 \mathrm{~mm}$. For the mustard seeds, which were approximately $2 \mathrm{~mm}$ in diameter, the slot orifices were $14 \mathrm{~mm}$ in width. One image of the flowing mustard seed is shown in Figure 1. Not visible in the figure is a larger filling silo above the experimental zone which keeps refilling the lower test silo.

Figure 2 displays resultant velocities of the four experiments (two particle types, two orifice geometries). Plotted in the figure is the norm of the velocity vector at five different heights above the exit orifice/s. Apparent in the figures is that close to the orifice the velocity appears to be Gaussian-like, in agreement with other studies. How- ever, further from the orifice, the velocity in the center of the silo is rather in-variant in the horizontal direction and plug-like. This is particularly evident in the double slot amaranth experiment, where the velocity far from the orifice is constant between two wall shear zones. It also appears that one effect of adding two adjacent orifices is to bring the plug flow zone closer to the orifice since, for both particle types, the flow $110 \mathrm{~mm}$ above the orifice is Gaussian-like in the single slot case, but more plug-like in the double slot case. Additionally, there is slip flow at the silo walls for the mustard seeds, but no flow at the walls was visible for the amaranth grains.

\section{Numerical model and results}

Recent experiments to measure the rheology of dense granular flow [24] allow us to model the bulk friction of granular materials using the following expression

$$
\mu(I)=\mu_{1}+\frac{\mu_{2}-\mu_{1}}{\frac{I_{0}}{I}+1}
$$

where $\mu_{1}$ and $\mu_{2}$ are the lower and upper limits of friction, $I_{0}$ is the inertial number when the friction is half way between $\mu_{1}$ and $\mu_{2}$, and $I$ is the granular inertial number, a function of the particle diameter, $d$, shear rate, $\dot{\gamma}$, particle density, $\rho_{p}$, and confining pressure, $P$;

$$
I=\frac{d|\dot{\gamma}| \sqrt{\rho_{p}}}{\sqrt{P}},
$$

By assuming that the volume fraction changes are small, that the deviatoric stress tensor aligns with the strain rate tensor, Jop [28] defined an effective granular viscosity as

$$
\eta(|\dot{\gamma}|, P)=\mu(I) P /|\dot{\gamma}| .
$$

We note that the yield using Equation 1 is a DruckerPrager criterion; material will not shear unless the shear stress is greater than the friction multiplied by the pressure. This effective viscosity has been successfully implemented in a Navier-Stokes solver (Gerris Flow Solver [25]) to model dense granular flow in various geometries $[15,16,29]$ including silo flow. In these works the viscosity was regularised using the following relation (which we also utilise in this work)

$$
\eta=\min \left(\frac{\mu(I) P}{D_{2}}, \eta_{\max }\right),
$$

where $D_{2}$ is the second invariant of the strain rate tensor.It was shown $[15,16]$ that such a model could account for the Beverloo scaling of the flow rate as a function of orifice width, and the behaviour was well matched with contactdynamics simulations.

In this investigation, a 2D model was solved using a similar method as in literature $[15,16]$. We do not model the flow as a two-fluid flow (that is, we do not investigate the free-surface evolution), but rather, make the assumption that the silo is continuously full and the top of the system is at atmospheric pressure. This procedure allows 

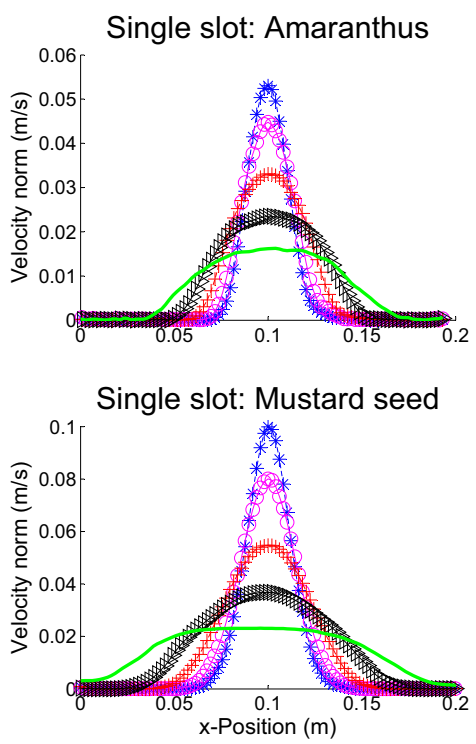

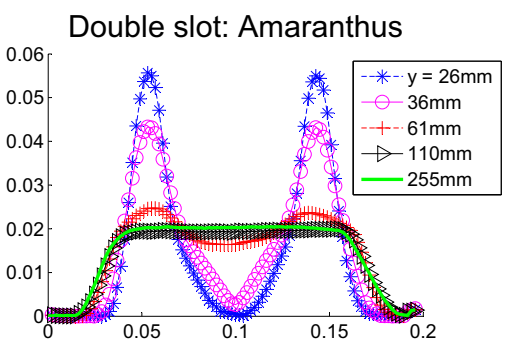

Double slot: Mustard seed

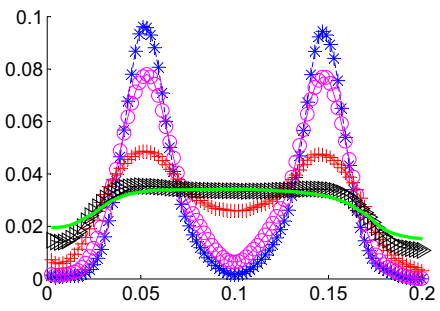

Figure 2. Experimental velocity curves at various heights above the silo orifice for single and two adjacent slot orifices (separated by $80 \mathrm{~mm}$ ), for two particle types, amaranth grains and mustard seeds.

friction at the side walls to be accounted for, but neglects the friction on the front (viewing side) and back of the silo walls. Although this is expected to have an effect, as a first investigation we neglect this aspect of the flow. The angle of repose of mustard seeds was found to be approximately $29.5^{\circ}$. Therefore, as an initial test of the friction parameters, we chose $\mu_{1}=\tan (29.5)$ degrees. The values of $I_{0}$ and $\mu_{2}$ were chosen to be $I_{0}=0.05$ and $\mu_{2}-\mu_{1}=0.25$. While these parameters are not measured, we found that they are reasonable approximations. We are pursuing experimental determination of the friction parameters using a set-up similar to Fall et al. [30]. The particle and bulk densities were given as, $\rho_{p}=1250 \mathrm{~kg} / \mathrm{m}^{3}, \rho_{b}=675 \mathrm{~kg} / \mathrm{m}^{3}$ and the particle diameter, $d=2 \mathrm{~mm}$. On the left and right walls the horizontal velocity was set to zero, and a Navier slip condition was applied for the vertical velocity with slip length $0.5 \mathrm{~d}=1 \mathrm{~mm}$. On the free surface and at the orifice the pressure was set to zero to simulate atmospheric conditions above and below the silo. The normal derivatives of the velocity components were set to zero above and below the silo. Solids with a no-slip condition were added near the orifice to model the position of the single and double orifice configurations.

Figure 3 displays velocity norm curves for the numerical mustard seed model to be compared with those of Figure 2. Near the orifice the Gaussian-like character of the flow is recovered, and the values of the speed are very close. Far from the orifice the flow is plug-like (although, the regularisation used slightly curves this zone). The addition of a second orifice has the effect of enlarging the plug-flow zone. In the figure, the red curve at $61 \mathrm{~mm}$ above the orifice is Gaussian-like in the single slot case, but pluglike in the double slot case. This was also seen in the experimental results of Figure 2 and indicates that the plug- like zone has extended closer to the bottom of the silo. Such results could be important to predict mixing in silo flows.

Qualitatively the behaviour is similar, yet is not perfect. The numerical model is more diffusive as we move further above the orifice. Far from the orifice the flowing zones are wider (and the shear zones where the velocity is changing rapidly less wide) in the numerical model than experimentally observed. In the numerical model the two orifices interact more strongly than was seen in the experiment. For example, in the center of the silo, at $x=0.1 \mathrm{~m}$, the interaction between the two orifices appears to be minimal in the experimental case as the velocity is almost zero at this location (for $y=26 \mathrm{~mm}$, profiles near the bottom of the silo). However in the numerical model this velocity is clearly non-zero, hence the interaction between the two orifices is still influencing the silo discharge. The slip velocity in the experiment was also seen to be larger than was predicted by the numerical model. This was particularly evident far from the orifice. Nevertheless, given that the friction of the front wall is neglected, and the rheological parameters and slip length at this stage remain best guesses, the results are encouraging. Once the friction parameters have been measured for our grains we plan to repeat the numerical tests with and without front wall friction.

\section{Discussion}

We have presented a first attempt to compare experimentally derived velocity fields using particle image velocimetry analysis, to results of a numerical model with a pressure-dependent viscosity, based upon the $\mu(I)$ granular rheology. We have shown that the numerical model 

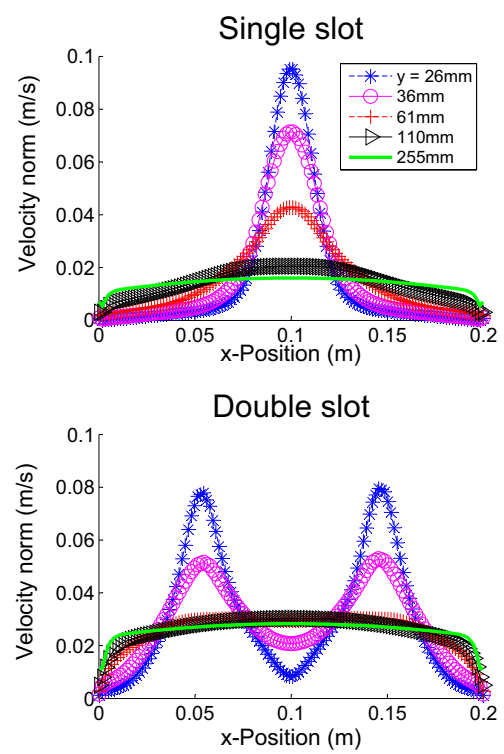

Figure 3. Numerical velocity curves generated using approximate parameters for mustard seed.

based on the $\mu(I)$ model is able to qualitatively capture the observed flow profiles in the silo, but the model is somewhat diffusive. The accurate resolution of plug-flow and Gaussian-like flow zones is critical to predict mixing, segregation, and residence time distributions precisely. Errors in the predicted velocity field will lead to errors in these measures and ultimately lessen the predictive capability of the model. This is particularly important in industries where quality control of granular flow is essential, such as the pharmaceutical and food industries. As such, further work is needed to accurately measure the flow parameters of the grains for the $\mu(I)$ model. The effect of friction on the front wall also needs to be investigated, as does other real-world effects such as cohesion between grain (perhaps generated by a high humidity environment).

However, we are encouraged by the preliminary behaviour of the model. With approximate parameters and several simplifications we have been able to reproduce many of the experimentally observed features of the flow.

In conclusion, the $\mu(I)$ model shows much promise to model industrial-scale granular flow and help in the understanding of granular flow phenomena.

\section{References}

[1] Y. Garfinkel, D. Ben-Shlomo, T. Kuperman, Antiquity 83, 309 (2009)

[2] W. Beverloo, H. Leniger, J. Van de Velde, Chemical engineering science 15, 260 (1961)

[3] C. Mankoc, A. Janda, R. Arevalo, J. Pastor, I. Zuriguel, A. Garcimartín, D. Maza, Granular Matter 9, 407 (2007)

[4] J. Choi, A. Kudrolli, M.Z. Bazant, Journal of Physics: Condensed Matter 17, S2533 (2005)
[5] L. Fullard, C. Davies, Applied Mathematical Modelling 40, 1494 (2016)

[6] L.A. Fullard, C.E. Davies, G.C. Wake, Advanced Powder Technology 24, 499 (2013)

[7] R.M. Nedderman, Statics and kinematics of granular materials (Cambridge University Press, 2005)

[8] G.J. Weir, Chemical Engineering Science 59, 149 (2004)

[9] F. Vivanco, F. Melo, C. Fuentes, International Journal of Bifurcation and Chaos 19, 3533 (2009)

[10] K. Kamrin, M.Z. Bazant, Physical Review E 75, 041301 (2007)

[11] K. Kamrin, C.H. Rycroft, M.Z. Bazant, Modelling and Simulation in Materials Science and Engineering 15, S449 (2007)

[12] R. Balevičius, R. Kačianauskas, Z. Mróz, I. Sielamowicz, Advanced Powder Technology 22, 226 (2011)

[13] R. Kobyłka, M. Molenda, Powder Technology 256, 210 (2014)

[14] Y. Yu, H. Saxén, Advanced Powder Technology 22, 324 (2011)

[15] L. Staron, P.Y. Lagrée, S. Popinet, Physics of Fluids (1994-present) 24, 103301 (2012)

[16] L. Staron, P.Y. Lagrée, S. Popinet, The European Physical Journal E 37, 1 (2014)

[17] I. Sielamowicz, S. Błoñski, T.A. Kowalewski, Chemical Engineering Science 61, 5307 (2006)

[18] I. Sielamowicz, A. Czech, T.A. Kowalewski, Powder Technology 270, 393 (2015)

[19] I. Sielamowicz, S. Blonski, T. Kowalewski, Chemical Engineering Science 60, 589 (2005)

[20] J. Gray, A. Thornton, Proceedings of the Royal Society of London A: Mathematical, Physical and Engineering Sciences 461, 1447 (2005)

[21] L. Fullard, C. Davies, Particuology 30, 102 (2017)

[22] K. Johanson, Powder technology 170, 109 (2006)

[23] X. Zhang, S. Zhang, G. Yang, P. Lin, Y. Tian, J.F. Wan, L. Yang, Physics Letters A 380, 1301 (2016)

[24] G. MiDi, The European Physical Journal E 14, 341 (2004)

[25] S. Popinet, Journal of Computational Physics 190, 572 (2003)

[26] W. Thielicke, E.J. Stamhuis, Journal of Open Research Software 2, e30 (2014)

[27] W. Thielicke, Ph.D. thesis, Ph. D. Thesis, Rijksuniversiteit Groningen (2014)

[28] P. Jop, Y. Forterre, O. Pouliquen, Nature 441, 727 (2006)

[29] P.Y. Lagrée, L. Staron, S. Popinet, Journal of Fluid Mechanics 686, 378 (2011)

[30] A. Fall, G. Ovarlez, D. Hautemayou, C. Mézière, J.N. Roux, F. Chevoir, Journal of Rheology (1978present) 59, 1065 (2015) 